\title{
Serum calprotectin level for diagnosis and detection of disease activity in rheumatoid arthritis
}

\author{
Nanees Adel $^{1}$, Marcel William ${ }^{1}$, Reham Al Swaff ${ }^{1, ~}$, Sherin Hassan ${ }^{2}$ \\ ${ }^{1}$ Internal Medicine Departments, Faculty of Medicine, Ain Shams University, Cairo, Egypt \\ ${ }^{2}$ Clinical Pathology Departments, Faculty of Medicine, Ain Shams University, Cairo, Egypt
}

\section{Email address:}

reham_ali_2004@hotmail.com (R. Al Swaff)

\section{To cite this article:}

Nanees Adel, Marcel William, Reham Al Swaff, Sherin Hassan. Serum Calprotectin Level for Diagnosis and detection of Disease Activity in Rheumatoid Arthritis. International Journal of Immunology. Vol. 2, No. 1, 2014, pp. 6-10. doi: 10.11648/j.iji.20140201.12

\begin{abstract}
Background: Early diagnosis of rheumatoid arthritis and timely detection of progression are global challenges. However, lack of sensitivity and imprecision of the currently available biomarkers have impaired the ability to implement potentially effective therapies in a timely manner. The present study aimed to evaluate the clinical utility of serum calprotectin in diagnosis as well as assessment of rheumatoid arthritis activity. Methods: serum calprotectin levels were measured in 60 Egyptian patients with rheumatoid arthritis (35 patients with active rheumatoid arthritis vs. 25 patients with quiescent disease) and 20 healthy subjects who served as a control group. Results: Serum calprotectin showed a highly significant elevation in patients with rheumatoid arthritis. Moreover, its level showed a highly significant increase during disease activity. Significant positive correlations were found between serum calprotectin and other markers of disease activity (ESR, CRP, WBCs, and platelets). Serum calprotectin at a cut- off level of $450 \mathrm{ng} / \mathrm{mL}$, had $75 \%$ sensitivity $\& 90 \%$ specificity for diagnosis of rheumatoid arthritis. Optimum cut-off level of calprotectin for prediction of disease activity was $950 \mathrm{ng} / \mathrm{mL}$ with $80 \%$ sensitivity, $76 \%$ specificity. Conclusion: serum calprotectin is a promising marker for diagnosis and monitoring of disease activity in patients with rheumatoid arthritis.
\end{abstract}

Keywords: Calprotectin, Rheumatoid, Arthritis, CRP

\section{Introduction}

Rheumatoid arthritis (RA) is a systemic auto-immune disease, with a main characteristic of persistent joint inflammation with substantial irreversible joint damage [1]. Around the world, one in every 100 persons suffers from RA. However, these figures may be underestimated since patients with mild disease may never seek medical opinion [2].

The clinical diagnosis of RA depends on the patient's clinical presentation and imaging techniques. However, imaging techniques show relative insensitivity to early bone damage with total insufficiency for assessment of soft tissue changes [3]. Many laboratory tests are included in the diagnosis as well as the early prediction of disease activity. In most patients with RA, the results of common laboratory tests as rheumatoid factor (RF), ESR, and C-reactive protein (CRP) levels are abnormal and may be used to support the diagnosis [4].

RF has been commonly used as a serological marker for RA; Although RF had a tolerable sensitivity of $75.9 \%$ for RA, but it had a low specificity of $78.7 \%$. Laboratory tests for prediction of disease activity include erythrocyte sedimentation rate (ESR), C- reactive protein (CRP), platelet and leucocyte count [5]. However, a substantial proportion of patients with RA have normal test results [4]. In addition, the sensitivity and specificity of these tests for changes in synovial inflammation are limited [6].

Calprotectin is a calcium-binding protein secreted predominantly by neutrophils and monocytes. Calprotectin is specifically released during interaction of monocytes with inflammatory activated endothelium, probably at site of local inflammation [7]. Calprotectin has been described in synovial tissue in RA patients', where it was found in the lining layer adjacent to the cartilage-pannus junction, which is the primary site of cartilage destruction and bone erosion [8]. Calprotectin concentration in diseased synovial fluid is 10 -fold higher than concentration in serum obtained in parallel from individual patients. Elevated calprotectin serum concentration therefore reflects release of these proteins from activated phagocytes within the synovium and the synovial fluid [9]. The resulting increase of calprotectin serum concentrations may be an important serum marker of the extent of local inflammation in 
the affected joints $[10,8]$.

The present study aimed to evaluate the clinical utility of serum calprotectin in diagnosis as well as assessment of RA activity.

\section{Subjects and Methods}

This study was conducted in the Internal Medicine and Clinical Pathology Departments, Ain Shams University Hospitals, Cairo, Egypt. 80 adult Egyptian subjects were recruited from the internal medicine and rheumatology outpatient clinics' and were classified into 3 groups as follows:

Group I: This group included 60 RA patients'. They were 7 males and 53 females. Their ages ranged from 20 to 58 year with a mean age of $39.3 \pm 8.6$ years. RA was diagnosed according to the American College of Rheumatology (ACR) criteria [11]. Patients were further classified according to disease activity described by Fransen et al. 2003 [12] into:

Subgroup Ia: This group included 35 RA patients' in activity. They were 3 males and 32 females. Their ages ranged between 20-55 years with a mean age of $38.9 \pm 8.60$ years. The majority of these patients were newly diagnosed.

Subgroup I b: This group included 25 RA patients' who were in clinical remission. They were 4 males and 21 females. Their ages ranged between $25-58$ years with a mean age of $39.9 \pm 8.9$ years. These patients had RA of $\geq 5$ years duration and all were receiving analgesics \pm corticosteroids \pm methotrexate for control of their illness.

Group II: This group included twenty age- and sexmatched healthy subjects serving as a control group. Their ages ranged between 25 to 49 years with a mean age of $36.2 \pm 6.5$ years.

All subjects included in the study were subjected to the following: history taking \&thorough clinical examination laying stress on number of tender \&/or swollen joints, X-Ray films of the affected joints for diagnosing structural joint damage, abdominal ultrasonography, laboratory investigations including: liver function tests, renal function tests, complete blood count (CBC), ESR, RF, CRP, urine analysis and Pregnancy test for female participants.

Serum calprotectin level measurement: The assay was carried out using Phical calprotectin ELISA kit (Immunodiagnostik AG, Stubenwald-Alee 8a, D64625 Bensheim).

Patients were excluded from study if they had any of the following conditions: any other autoimmune disorder (i.e.: inflammatory bowel disease, pernicious anaemia, multiple sclerosis), acute or chronic kidney disease, any form of current acute or chronic infection, any form of chronic decompensated disease, organ transplant recipient, current or past history of any malignancy, Felty's syndrome, patients receiving any form of biological therapy, pregnant or nursing females.

This study was approved by the local ethical committee of the Ain Shams University hospitals and a written informed consent was obtained from each participant.

\subsection{Statistical Methods}

Statistical analysis was done using software SPSS version 16. Data were described as mean $(\overline{\mathrm{X}})$ and standard deviation (SD) in case of parametric data $\&$ as median and the intrequartile range in case of skewed data. The following tests were applied: The Student's t test, Mann-Whitney U test (Wilcoxon's rank sum test [z]) and Spearman's rank correlation coefficient $\left(\mathrm{r}_{\mathrm{s}}\right)$. P value $>0.05$ considered non-significant, $\mathrm{p}<0.05$ considered significant $\& \mathrm{p}<0.001$ considered highly significant. Sensitivity, specificity, diagnostic efficiency, positive \& negative predictive value of each test were calculated $\&$ the overall diagnostic performance of a test was assessed by receiver- operating characteristics (ROC) curve analysis.

\section{Results}

The current study revealed a highly significant increase in serum calprotectin level when comparing active RA patients' with controls $(\mathrm{p}<0.001)$, inactive RA patients' with controls $(p<0.001)$, active RA patients' with inactive RA patients' $(\mathrm{p}<0.001)$ and when comparing all patients to controls ( $\mathrm{p}<0.001)$ (Table 1$)$.

Correlation study between serum calprotectin level and other studied parameters in RA patients' revealed a highly significant positive correlation between serum calprotectin level and other markers of disease activity (ESR, white blood cells (WBCs), platelets). Also there was a significant positive correlation between serum calprotectin level and CRP as well as significant negative correlation between serum calprotectin and hemoglobin level $(\mathrm{Hb})$. A non- significant correlation was found between serum calprotectin and RF levels (Table 2).

Table 1. Comparison between the different studied groups regarding serum calprotectin levels.

\begin{tabular}{ccc}
\hline Compared Group & \multicolumn{2}{c}{ s. calprotectin $(\mathbf{n g} / \mathbf{m L})$} \\
\hline & $\mathrm{z}$ & $\mathrm{p}$ \\
Inactive RA vs. control & -3.11 & $<0.001$ \\
Active RA vs. control & -5.55 & $<0.001$ \\
Inactive RA vs. Active RA & -4.3 & $<0.001$ \\
All Patients vs. Control & -5.04 & $<0.001$ \\
\hline
\end{tabular}

Table 2. correlation between serum calprotectin and other studied parameters in RA Patients,

\begin{tabular}{ccc}
\hline calprotectin(ng/mL) & $\mathbf{r}_{\mathbf{s}}$ & $\mathbf{p}$ \\
$\mathrm{ESR}(\mathrm{mm} / \mathrm{h})$ & 0.486 & $<0.001$ \\
$\mathrm{CRP}(\mathrm{mg} / \mathrm{L})$ & 0.406 & $<0.05$ \\
$\mathrm{Hb}(\mathrm{gm} / \mathrm{dL})$ & -0.285 & $<0.05$ \\
$\mathrm{WBC}\left(\mathrm{x} 10^{3} / \mathrm{cmm}\right)$ & 0.462 & $<0.001$ \\
$\mathrm{Platelets}\left(\times 10^{3} / \mu \mathrm{L}\right)$ & 0.524 & $<0.001$ \\
$\mathrm{RF}(\mathrm{IU} / \mathrm{mL})$ & 0.043 & $>0.05$ \\
\hline
\end{tabular}

Serum RF value had $80 \%$ sensitivity but only $20 \%$ specificity and $55 \%$ diagnostic efficacy in discriminating patients with active RA from those with inactive disease while CRP was more accurate in discrimination of RA activity with $100 \%$ sensitivity, $88 \%$ specificity and overall efficacy of $95 \%$ (Table 3 ).

Receiver-operating characteristic (ROC) curve analysis 
was applied to determine the best cut-off value of serum calprotectin in diagnosing RA. The Area under the curve (AUC) was 0.863 , and the optimum cut-off level was $450 \mathrm{ng} / \mathrm{mL}$ (Figure 1). This had a diagnostic sensitivity, specificity, negative predictive value, positive predictive value and efficacy of $75 \%, 90 \%, 54.55 \%, 95.74 \%$ and $78.75 \%$ respectively (Table 4 ).
When assessing serum calprotectin value in discriminating patients with active RA from those with quiescent disease, the AUC was 0.754 and the optimum cut-off level was $950 \mathrm{ng} /$ $\mathrm{mL}$ (Figure 2). This had diagnostic sensitivity, specificity, negative predictive value, positive predictive value and overall efficacy of $80 \%, 76 \%, 73.08 \%, 82.35 \%$ and $78.33 \%$ respectively (Table 5).

Table 3. diagnostic performance of $R F$ and $C R P$ in patients with active $R A v$ s. patients with inactive disease.

\begin{tabular}{cccccc}
\hline Parameter & Sensitivity(\%) & Specificity(\%) & $\begin{array}{c}\text { Negative Predictive } \\
\text { Value(\%) }\end{array}$ & $\begin{array}{c}\text { Positive Predictive } \\
\text { Value(\%) }\end{array}$ & Efficacy(\%) \\
\hline $\mathrm{RF}(\mathrm{IU} / \mathrm{mL})$ & 80 & 20 & 41.7 & 58.3 & 55 \\
$\mathrm{CRP}(\mathrm{mg} / \mathrm{L})$ & 100 & 88 & 100 & 92.1 & 95 \\
\hline
\end{tabular}

Table 4. diagnostic performance of serum calprotectin level in RA patients'vs. the control group.

\begin{tabular}{rcccccc}
\hline Parameter & $\begin{array}{c}\text { ROC } \\
\text { Cut-off }\end{array}$ & $\begin{array}{c}\text { Sensitivity } \\
(\%)\end{array}$ & Specificity (\%) & $\begin{array}{c}\text { Negative } \\
\text { Predictive } \\
\text { Value(\%) }\end{array}$ & $\begin{array}{c}\text { Positive } \\
\text { Predictive } \\
\text { Value(\%) }\end{array}$ & $\begin{array}{c}\text { Diagnostic } \\
\text { Efficacy(\%) }\end{array}$ \\
\hline s.calprotectin $(\mathrm{ng} / \mathrm{mL})$ & 450 & 75 & 90 & 54.44 & 95.74 & 78.75 \\
\hline
\end{tabular}

Table 5. diagnostic performance of serum calprotectin $(\mathrm{ng} / \mathrm{mL})$ in patients with active RA vs. patients with inactive disease.

\begin{tabular}{cccccc}
\hline Parameter & $\begin{array}{c}\text { ROC Cut- } \\
\text { off }\end{array}$ & $\begin{array}{c}\text { Sensitivity } \\
(\%)\end{array}$ & $\begin{array}{c}\text { Specificity } \\
(\%)\end{array}$ & $\begin{array}{c}\text { Negative } \\
\text { Predictive } \\
\text { Value (\%) }\end{array}$ & $\begin{array}{c}\text { Positive } \\
\text { Predictive } \\
\text { Value (\%) }\end{array}$ \\
\hline s.calprotectin(ng/mL) & 950 & 80 & 76 & $\begin{array}{c}\text { Diagnostic } \\
\text { Efficacy (\%) }\end{array}$ \\
\hline
\end{tabular}

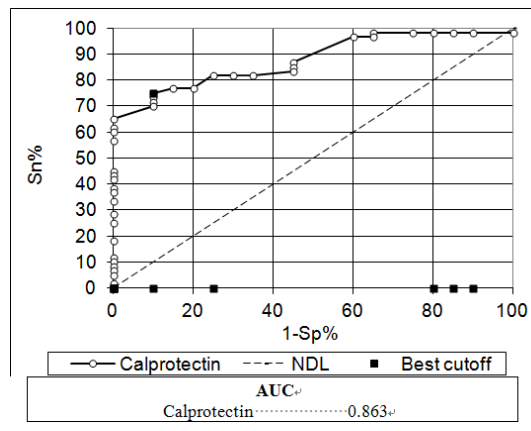

Figure (1). ROC curve analysis showing the diagnostic performance of calprotectin for discriminating patients with RA from the control group.

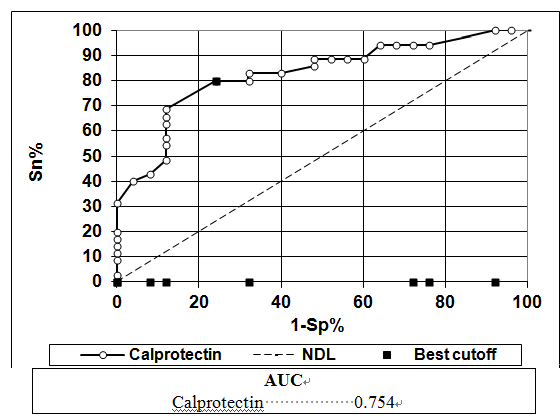

Figure (2). ROC curve analysis showing the diagnostic performance of calprotectin for discriminating patients with active RA from patients with inactive disease.

\section{Discussion}

RA is a chronic inflammatory autoimmune disease characterized by synovitis and joint destruction in which the infiltration of inflammatory cells, the activation of synovial fibroblasts and the production of a wide range of inflammatory mediators play significant roles [13].

During the past two decades there has been increasing interest in calprotectin, a major leucocyte protein, which constitutes about $40-60 \%$ of the soluble cytosolic protein content in neutrophilic granulocytes, as well as being a major monocyte/macrophage protein. Calprotectin is released during the interaction of monocytes with inflammatory activated endothelium, and it binds to endothelial cells and modulates transendothelial migration of leucocytes [8].

The current study revealed a highly significant elevation of serum calprotectin level in patients with RA. This goes in agreement with Chen et al. 2009 [6] who found higher levels of calprotectin in patients with RA and this was in keeping with the high calprotectin gene expression reported in patients with such a disease [6].

In the present study, serum calprotectin showed highly significant higher levels in patients with active RA when compared to patients with quiescent disease. This finding goes in agreement with many previous studies which stated that the association between calprotectin and joint damage is biologically plausible as during inflammation, both macrophages and granulocytes are recruited into the synovium and synovial fluid. These cells contain large amounts of calprotectin, which is released during activation and cell death. The protein is small, and can pass easily into the circulation thus calprotectin levels can reflect the degree of inflammatory activity in the joints [10, 14]. Studies on serum level of calprotectin in patients with RA have confirmed an excellent correlation of serum calprotectin concentration with the inflammatory activity of arthritis [6]. 
In clinical practice, laboratory parameters most frequently used in the monitoring of disease activity, in chronic arthritis, are CRP and ESR levels. The present study revealed significant positive correlations between serum calprotectin and both ESR and CRP in RA patients'. This finding is in accordance with De Seny et al. 2008 [15] and Hammer et al. 2010 [16] who reported that calprotectin may behave like an acute phase protein. However an improved face validity of calprotectin as a marker is that the protein is released from activated leucocytes which are derived mainly from the inflamed synovium in patients with RA. In contrast, the acute phase proteins CRP and ESR are primarily produced in hepatocytes after induction by interleukins released during inflammation. Thus calprotectin is a more advantageous marker compared to acute phase proteins by directly reflecting the amount of activated leucocytes in the inflamed joints [16].

The present study also revealed highly significant positive correlations between serum calprotectin and both WBCs and platelets in patients with RA. Similar findings were previously confirmed by Brun et al. 1995[17]. This can be mostly attributed to the fact that calprotectin constitutes about $40-60 \%$ of the soluble cytosolic protein content in neutrophilic granulocytes as well as being a major monocyte/macrophage protein.

A remarkable finding of the current study is the non significant correlation between serum calprotectin and RF levels. Although this is opposite to the finding of Hammer et al.2008 [10] who reported a significant positive correlation between the two markers, other investigators denoted that calprotectin is not correlated with IgM RF and explained this by the stronger association of calprotectin with the disease activity rather than with the autoimmune response [8].

The present study evaluated the possibility of using serum calprotectin to discriminate patients with active RA from those with inactive disease. At a cut off level of 950 $\mathrm{ng} / \mathrm{mL}$ with AUC of 0.754 , the diagnostic sensitivity was $80 \%$; specificity was $76 \%$ with an overall accuracy of $78.3 \%$. To our knowledge, Hammer et al .2010 [16] was the only study which evaluated the performance of calprotectin in discriminating patients with progressive radiographic joint damage from those with quiescent disease and reported a cut off level of calprotectin at $1.86 \mathrm{mg} / \mathrm{L}$ to have a diagnostic sensitivity of $69 \%$ and specificity of $66 \%$.

Concerning the diagnostic performance of RF in patients with active RA versus patients with inactive disease, the diagnostic sensitivity was $80 \%$, the diagnostic specificity was $20 \%$ and the diagnostic accuracy was $55 \%$. CRP proved to have a better diagnostic performance in discriminating patients with active RA from those with inactive disease with a diagnostic sensitivity of $100 \%$, specificity of $88 \%$, and an overall accuracy of $95 \%$. Although calprotectin is proved to be a promising marker in diagnosis of disease activity, yet CRP remains more sensitive than calprotectin as a marker of disease activity. However, it seems logic to suspect that calprotectin will be more accurate on follow up of disease activity in patients receiving biological therapy as biological drugs against the cytokines TNF $\alpha$, interleukin (IL) 1 or IL6 will reduce the circulating cytokines and thus the concentration of the acute phase proteins. Calprotectin, however, is not directly influenced by these drugs, and will only reflect the leucocyte activation during inflammation [16].

According to all previous results, it is recommended to consider serum calprotectin as a new diagnostic and screening tool in RA in order to improve the ability to identify such patients and thus could allow the earlier initiation of treatment. The expected value of calprotectin in follow up of patients on biological therapy needs to be evaluated.

The present clinical trial had few limitations. First, calprotectin level was not measured in the synovial fluid. However, the protein is small and can pass easily into the circulation thus serum calprotectin level can reflect the degree of inflammatory activity in the joints [10, 14]. Second, the correlation between serum calprotectin level and the degree of radiographic joint damage was not evaluated. Third, the number of patients included in the present study is relatively small.

\section{Conclusion}

Serum calprotectin is a promising marker for diagnosis and monitoring of disease activity in patients with RA.

\section{Disclosure}

The authors have no financial disclosures or conflicts of interest to declare.

\section{References}

[1] Helmick C.G., Felson D.T., Lawrence R.C., et al. Estimates of the Prevalence of Arthritis and other Rheumatic Conditions in the United States: Part I. Arthritis Rheum 2008; 58(1):15-25.

[2] Visser H. Early Diagnosis of Rheumatoid Arthritis. Best Practice and Research Clinical Rheumatology 2005; 19 (1): 155-172.

[3] Ostergaard M., Ejbjerg B., Szkudlarek M. Imaging in Early Rheumatoid Arthritis: Roles of Magnetic Resonance Imaging Ultrasonography, Conventional Radiography and Computed Tomography. Best Practice and Research Clinical Rheumatology 2005; 19 (1): 91-116.

[4] Sokka T. and Pincus T.. Erythrocyte Sedimentation Rate, C reactive protein, or Rheumatoid Factor are Normal at Presentation in $35 \%-45 \%$ of Patients with Rheumatoid Arthritis Seen between 1980 and 2004: Analyses from Finland and the United States. J Rheumatol 2009; 36: 1387-1390.

[5] Riel P. and Fransen J. Established Rheumatoid Arthritis: Clinical Assessments. Best Practice and Research Clinical Rheumatology 2007; 21 (5): 807-825.

[6] Chen Y.S., Yan W., Geczy C.L., et al. Serum levels of soluble receptor for advanced glycation end products and of S100 proteins are associated with inflammatory, autoantibody, and classical risk markers of joint and vascular damage in rheumatoid arthritis. Arthritis Res Ther. 2009; 11:R39. 
[7] Hammer H.B., Odegard S., Fagerhol M.K. Calprotectin (a major leucocyte protein) is strongly and independently correlated with Joint Inflammation and Damage in Rheumatoid Arthritis. Ann Rheum Dis. 2007; 66:1093-1097.

[8] Andrés Cerezo L., Mann H., Pecha O., et al. Decreases in serum levels of S100A8/9 (calprotectin) correlate with improvements in total swollen joint count in patients with recent-onset rheumatoid arthritis. Arthritis Res Ther. 2011; 13(4):R122.

[9] Foell D., Wittkowski H.,Vogl T., et al. S100 proteins expressed in phagocytes: a novel group of damageassociated molecular pattern molecules .Journal of Leukocyte Biology 2007; 81: 28-37.

[10] Hammer H.B., Haavardsholm E.K. , Kvien T.K. Calprotectin (a major leucocyte protein) is associated with the levels of anti-CCP and rheumatoid factor in a longitudinal study of patients with very early rheumatoid arthritis. Scand J Rheumatol 2008; 37:179-182.

[11] Aletaha D., Neogi T., Silman A.J. 2010 rheumatoid arthritis classification criteria: an American College of Rheumatology/European League against Rheumatism collaborative initiative. Ann. Rheum. Dis. 2010; 69 (9): $1580-1288$

[12] Fransen J., Stucki G., van Riel L.C.M. Rheumatoid Arthritis Measures Disease Activity Score (DAS), Disease Activity
Score-28 (DAS28), Rapid Assessment of Disease Activity in Rheumatology (RADAR), and Rheumatoid Arthritis Disease Activity Index (RADAI) Arthritis \& Rheumatism. Arthritis Care \& Research 2003; 49(5): 214 -224.

[13] Tobón G.J., Youinou P., Saraux, A.The environment, geoepidemiology, and autoimmune disease: Rheumatoid arthritis. JAutoimmun. 2010; 35(1):10-14.

[14] Madland T.M., Hordvik M., Haga H.J., et al. Leukocyte protein calprotectin and outcome in rheumatoid arthritis. Scand J Rheumatol $2002 ; 31: 351-354$.

[15] De Seny D., Fillet M., Ribbens C., et al. Monomeric Calgranulins Measured by SELDI-TOF Mass Spectrometry and Calprotectin Measured by ELISA as Biomarkers in Arthritis. Clinical Chemistry 2008; 54: 1066-1075.

[16] Hammer H.B., Odegard S., Syversen S.W., et al. Calprotectin (a major S100 leucocyte protein) predicts 10year radiographic progression in patients with rheumatoid arthritis. Ann Rheum Dis.2010; 69:150-154.

[17] Brun JG, Nilssen S, Kvale G. Breast feeding, other reproductive factors and rheumatoid arthritis. A prospective study. Br J Rheumatol 1995;34:542-6. 